\title{
OBSTETRIC AND PSYCHOLOGICAL CARE FOR PREGNANT WOMAN WITH HYPEREMESIS GRAVIDARUM WITH MODERATE ANXIETY
}

\author{
Hesti Yuniastutiningsih"; Dini Latifatun Nafi'ati² \\ Correspondence: hesti.reddi@gmail.com \\ ${ }^{1}$ RSUD Tamiang Layang Kabupaten Barito Timur Kalimantan Tengah, Indonesia; ${ }^{2}$ SMF Psikiatri RSUD Dr. Saiful Anwar Malang, Indonesia
}

\section{ABSTRACT}

Physical and psychological changes experienced by pregnant women, especially hormonal changes, cause symptoms of nausea and vomiting in early pregnancy. Excessive nausea and vomiting in early pregnancy and persist throughout pregnancy will cause physical and psychological complications. The existence of psychological problems in pregnant women can predispose to nausea and vomiting which then worsens. Emotional problems are also associated with the incidence of nausea and vomiting becoming more severe. Methods: the authors conducted extensive searches by scientific journals through trusted and frequently used databases, namely PubMed, Springer, and Science Direct. The keywords used were "hyperemesis" AND "psychological" with journal publication filters, the last 4 years, randomized clinical trials, systematic reviews, meta-analysis, human research subjects. Conclusion: care that focuses on mothers by applying the principles of holistic care, where patients not only receive midwifery care physically and biologically but include psychological, social, spiritual and cultural by involving their husbands in midwifery care is proven to accelerate the physical and psychological recovery of hyperemesis sufferers.

Keywords: hyperemesis, psychological, anxiety.

\section{Article History:}

Received: April 6, 2021

Accepted: July 5, 2021

Published: September 30, 2021
Cite this as: Yuniastutiningsih, H. Nafi'ati, D.N. Obstetric and psychological care for pregnant woman with hyperemesis gravidarum with moderate anxiety. Journal of Psychiatry Psychology and Behavioral Research; 2021. Vol. 2, Issue 2. p: 11-13.

\section{INTRODUCTION}

Physical and psychological changes experienced by pregnant women, especially hormonal changes that cause symptoms of nausea and vomiting in early pregnancy. Nausea and vomiting during pregnancy affect most women with varying incidence rates, ranging from $35 \%$ to $91 \%$ with a mean of $69 \%$ with varying severity. ${ }^{1}$ Excessive nausea and vomiting in early pregnancy and persist throughout pregnancy will cause physical and psychological complications. Psychologically, many women feel that nausea and vomiting affect quality of life, contribute to high levels of depression, can strain family relationships, and become an economic burden. ${ }^{2}$

Recent literature suggests a genetic predisposition in addition to previously studied factors such as infection, psychiatry, and hormonal contributions as triggers of hyperemesis garvidarum, although research is continuing to find its etiology. Impacts on maternal morbidity are frequent and include psychological effects, financial burdens, clinical complications of nutritional deficiency, gastrointestinal trauma, and, in rare cases, neurological damage. ${ }^{3}$
The association of hyperemesis gravidarum with the psychological state of pregnant women has been suggested by several studies that found an increased risk of hyperemesis gravidarum in women with a history of depression, while other studies found an increased risk of depression in women with hyperemesis gravidarum who had no history of psychiatric illness. ${ }^{4}$

The existence of psychological problems in pregnant women can predispose to nausea and vomiting which then worsens. Emotional problems are also associated with the incidence of nausea and vomiting becoming more severe. Psychological factors that may have an effect are anxiety, stress, or depression. This is a reaction from the body in dealing with unusual situations, such as situations that endanger, worry, and confuse someone. ${ }^{2}$

In addition to increasing morbidity, hyperemesis gravidarum also has long-term effects that affect the patient's psychology. One study suggested that women with hyperemesis gravidarum were more likely to report emotional distress than women without hyperemesis gravidarum during pregnancy and 6 months postpartum. ${ }^{4}$ 
Treatment of hyperemesis gravidarum, which focuses not only on the patient's physical needs, but also on his psychological needs, is expected to accelerate the patient's healing and prevent long-term impacts on the patient's psychology. Therefore, holistic midwifery care, including bio-psychosocio-cultural, is needed by pregnant women with hyperemesis gravidarum to accelerate recovery and prevent complications.

\section{CASE}

Woman, 32 y.o, junior high school graduate, housewife, working as a rubber tapper before becoming pregnant. Her husband, 38, is an employee of the Credit Union cooperative, which belongs to a Catholic Foundation. Having one child aged 8 years, during the pregnancy of the first child, the mother experienced excessive nausea and vomiting, so that during the pregnancy of the first child the mother was hospitalized 7 times. The birth of the first child was carried out by SC on the indication that the mother was weak due to the hyperemesis she suffered so that she was unable to push. This pregnancy was not planned, because the mother was traumatized during her first pregnancy which was felt to be very heavy due to nausea, vomiting and inability to carry out normal activities. The mother used regular abstinence for 8 years because she could not use contraceptive pills and injections (always nausea and vomiting). Since the beginning of this pregnancy, the patient experienced excessive nausea and vomiting until she was unable to carry out daily activities. Patient often cried and felt unable to undergo this pregnancy because of this, often feels frightened by her own thoughts, feels a lump in her neck and her feelings change throughout the day.

Chronological flow of moderate anxiety experienced by patient due to her pregnancy was not planned, trauma with a history of previous pregnancy and stigma of neighbors in the area about hyperemesis. Objective data revealed general condition of the mother is good, compos mentis awareness, with vital signs: temperature $36.2{ }^{\circ} \mathrm{C}$, pulse $100 \mathrm{x} /$ minute, blood pressure 100/60 $\mathrm{mmHg}$, breathing 24 times / minute. Height $148 \mathrm{~cm}$, weight before pregnancy $55 \mathrm{~kg}$, current weight $49 \mathrm{~kg}$, upper arm circumference $24 \mathrm{~cm}$. Physical examination from head to toe found no abnormalities. The psychological assessment found a 22 / moderate anxiety scale (HARS assessment). Intervention to this patient such as midwifery, psychological and community implementation intervention.

Evaluation after intervention revealed that patient in good condition after she felt already listened by counselor and got family and her environment support.

\section{METHOD}

The authors conducted extensive searches by scientific journals through trusted and frequently used databases, namely PubMed, Springer, and Science Direct. The keywords used were "hyperemesis" AND "psychological" with journal publication filters, the last 4 years, randomized clinical trials, systematic reviews, meta-analysis, human research subjects.

\section{RESULT}

Hyperemesis gravidarum (HG) is a condition in which the patient experiences excessive nausea and vomiting, in such a way as to interfere with the patient's activities and overall health. ${ }^{5}$

In all publications, negative themes of hyperemesis gravidarum have been reported. The 2017 Norwegian study ${ }^{4}$ reported that women with $\mathrm{HG}$ had a higher chance of experiencing emotional distress than women without $\mathrm{HG}$ at 17 weeks' gestation $(p<0.001)$ and 32 weeks of gestation $(p=$ $0.001)$ and 6 months postpartum $(p=0.005)$. The adjusted odds for emotional distress varied significantly over time for women with and without HG $(p=0.035)$.

Other research has shown that the psychosocial burden of hyperemesis may be underestimated. A survey by Poursharif et al. ${ }^{6}$ described the characteristics of women who terminated their pregnancies due to hyperemesis gravidarum (HG), patients suffering from hyperemesis gravidarum were more likely to report that their health care providers were unaware of how sick they were. Of the 808 women with hyperemesis studied, $15 \%$ had at least one termination because of their symptoms, and another $12 \%$ "nearly" had terminated a pregnancy. Many patients with hyperemesis report fear of a subsequent pregnancy because of their experience with hyperemesis. The patient-doctor relationship may be strained by the caregiver's attitude to hyperemesis, as some relate hyperemesis as a manifestation of psychosocial stress or negative attitudes toward pregnancy. ${ }^{6}$

Emotional stress caused by hyperemesis and the assumption that hyperemesis is a manifestation of stress experienced by sufferers, clarifies the close relationship between psychological factors and hyperemesis. The importance of the combination of intravenous fluid administration with psychological interventions in accelerating recovery was stated by Jinjuan YU and Xingping PAN in $2017^{7}$ who suggested that there was a difference in the average days of stay in the group of hyperemesis sufferers who were given nutritional solutions intravenously and routine care on an average basis. The mean days of hospitalization were (7.4 \pm 0.4 ) days, while the group of hyperemesis patients who had intravenous nutritional fluids combined with psychological intervention had a mean of days of hospitalization $(5.3 \pm 0.6)$ days. From the results of these studies it can be concluded that intravenous nutrition combined with psychological intervention in the treatment of hyperemesis gravidarum has a good effect, which can effectively increase the effect of clinical treatment, and reduce hospitalization days, and has a high clinical application value.

\section{DISCUSS}

Pregnancy is a period of change, adjustment, challenges and turning points in family life, and it has a profound impact on every aspect of women's life. Changes during pregnancy and childbirth occur both physically and psychologically.

The adaptation process in dealing with the physical and psychological changes of pregnancy becomes more difficult if the pregnancy is unexpected due to a bad experience in a previous pregnancy. The failure of the adaptation process can have a negative impact on pregnancy, both physically and psychologically, which is a mutually supporting phenomenon. The situation can be exacerbated by the existence of a conflict between desires that are grown from sociocultural norms and problems in pregnancy. This can lead to psychological problems that can occur due to a difficult pregnancy, immaturity in emotional and psychosexual development, 
repeated past trauma, and feeling unable to be a complete woman.

Anxiety about the continuity of pregnancy is one of the stressors in women with a history of hyperemesis gravidarum. As stated by Taylor (2018) anxiety is a subjective experience of disturbing mental tension as a general reaction and the inability to deal with problems or the presence of safety. ${ }^{8}$ According to Deffenbacher and Hazaleus ${ }^{8}$, there are several sources of anxiety, including worry which is negative thoughts about oneself, emotionality as a self-reaction to autonomic nervous stimuli such as heart palpitations and cold sweat, and disturbances and obstacles to completing tasks (task generated

\section{CONCLUSION}

Pregnancy is a period of change, adjustment, challenges and turning points of family life, as well as profoundly affecting every aspect of a woman's life. Changes during pregnancy and childbirth occur both physically and psychologically, which have an impact, including anxiety.

Excessive anxiety can lead to depression. Mothers who experience anxiety are caused by negative thoughts that shape the mother's perception, so it is necessary to take a cognitive approach to eliminate these negative thoughts and lead to positive thoughts in accordance with the existing reality, because the anxiety experienced by the mother will affect pregnancy, especially hyperemesis conditions. experienced. Support and attention from people around them, including midwifery service providers, will give mothers a feeling of comfort in dealing with physical and psychological changes during pregnancy, as well as being motivated to carry out their pregnancy well. Health professionals can help women by letting them express their feelings, and prove them. If needed, referral to the mental health team may be necessary.

Case of anxiety due to hyperemesis gravidarum and a sense of trauma in a previous pregnancy. After doing a cognitive psychological approach, the mother experienced a decrease in the HARS anxiety scale from a total score of 22 to a score of 13. Care that focuses on mothers by applying the principles of holistic care, where the patient does not only receive physical and biological midwifery care but includes psychological, social, spiritual and culture by involving husbands in midwifery care is proven to accelerate the physical and psychological recovery of sufferers of hyperemesis. Moral support and encouragement need to be given to the mother and convince the mother that she is able to undergo this pregnancy is recommended but must still refer to the health condition and also the next intervention plan to be carried out.

\section{REFERENCES}

1. Nicola Mitchell- Jones, Kim Lawson, Shabnam Bobdiwala, Jessica Alice Farren, Aurelio Tobias, Tom Bourne CB. Association between hyperemesis gravidarum and psychological symptoms, psychosocial outcomes and infant bonding: a two-point prospective case - control multicentre survey study in an inner city setting. BMJ. 2020

2. Rorrong JF, Wantania JJE, Lumentut AM. Psychological Relationship between Pregnant Women and the Incidence of Hyperemesis Gravidarum. e-CliniC. 2021; 9 (28): 218 23. interference) is a tendency experienced by someone who is always depressed because of rational thinking about the task. The existence of psychological problems in pregnant women can predispose to nausea and vomiting which then worsens. Emotional problems are also associated with the incidence of nausea and vomiting becoming more severe. Anxiety can aggravate hyperemesis or hyperemesis can cause anxiety which can lead to depression. Because of this close relationship, comprehensive midwifery care and treatment is needed, not only focusing on physical conditions but also covering biological, psychological, social and cultural needs.

3. Abulafia VLSGDMSO. Hyperemesis Gravidarum: A Review of Recent Literature. Pharmacology. 2017;: 161171.

4. Kjeldgaard HK, Eberhard-Gran M, Benth JŠ, Vikanes $\AA \mathrm{V}$. Hyperemesis gravidarum and the risk of emotional distress during and after pregnancy. Arch Womens Ment Health. 2017 Dec 1; 20 (6): 747-56.

5. Achadiat CM. Obstetrics and Gynecology. I. Jakarta: Book Medical Publishers EGC; 2004. 72 p.

6. Poursharif B, Korst LM, MacGibbon KW, Fejzo MS, Romero R, Goodwin TM. Elective pregnancy termination in a large cohort of women with hyperemesis gravidarum. Contraception. 2007; 76 (6): 451-5.

7. PAN JY; X. Analysis on the curative effect of intravenous nutrition liquid combined with psychological intervention on the treatment of the patients with hyperemesis gravidarum. Chinese J Biochem Pharm 2017; 37 (8) 1718. 2017;

8. Azis NAM. Coping Strategies Against Anxiety in Pregnant Women with a History of Miscarriage in Previous Pregnancy. J Ilm of Applied Psychology. 2017; 05, No. 1: 144-57. 